\title{
Mitteilungen
}

\section{Schweizerische Arbeitsgemeinschaft für Laparo- und Thorakoskopische Chirurgie SALTC}

\section{Zollikofer-Preis}

Die Schweizerische Arbeitsgemeinschaft für Laparo- und Thorakoskopische Chirurgie SALTC fördert aktiv die Forschung in laparoskopischer und thorakoskopischer Chirurgie und verleiht 2009 erneut den Zollikofer-Preis von 3000 Franken für eine herausragende wissenschaftliche Arbeit auf diesem Gebiet.

Thema: Grundlagenforschung oder klinische Forschung im Bereich der laparoskopischen und thorakoskopischen Chirurgie.

Bedingungen: Die Arbeit muss im Jahre 2008 oder 2009 als Originalartikel publiziert oder zur Publikation akzeptiert sein. Schweizer Autoren können auch im Ausland erarbeitete Beiträge einsenden, ausländische Autoren können in der Schweiz entstandene Arbeiten einreichen. Sprachen: Englisch, Französisch, Deutsch.

\section{Einsendeschluss: 3. Mai 2009}

Einsenden an: PD Dr. D. Hahnloser, Wissenschaftliche Kommission SALTC, Klinik für Viszeral- und Transplantationschirurgie, UniverstätsSpital Zürich, 8091 Zürich, E-Mail: dieter.hahnloser@usz.ch

\section{Association Suisse pour la Chirurgie} Laparoscopique et Thoracoscopique ASCLT

\section{Prix «Zollikofer»}

L'Association Suisse pour la Chirurgie Laparoscopique et Thoracoscopique ASCLT met au concours le prix «Zollikofer», d'un montant de 3000 francs, pour l'encouragement de jeunes chercheurs.

Thème: Recherche de base ou clinique dans le domaine de la chirurgie laparoscopique ou thoracoscopique.

Conditions: Le travail de recherche doit être publié (article original) ou accepté pour publication en 2008ou 2009. Le travail peut être fait à l'étranger si l'auteur principal est Suisse.
Les auteurs étrangers doivent avoir accompli la recherche en Suisse. Langues: Anglais, Français, Allemand.

\section{Date limite des envois: 3 mai 2009}

Adresse: PD Dr. D. Hahnloser, Wissenschaftliche Kommission SALTC, Klinik für Viszeralund Transplantationschirurgie, UniverstätsSpital Zürich, 8091 Zürich, e-mail: dieter. hahnloser@usz.ch

\section{Swiss Association for Laparoscopic} and Thoracoscopic Surgery SALTS

\section{"Zollikofer" Young Investigators Award}

The Swiss Association for Laparoscopic and Thoracoscopic Surgery SALTS strongly supports research in laparoscopic and thoracoscopic surgery. The Association invites researchers to apply for the "Zollikofer" Young Investigators Award (CHF 3'000) of the year 2009.

Topic: Basic science and clinical investigation in laparoscopic and thoracoscopic surgery.

Requirements: Original article published in 2008 or 2009 or accepted for publication. Swiss authors can submit work done abroad, nonSwiss authors must have performed their research in Switzerland. Language: English, French or German.

Deadline: $3^{\text {rd }}$ May 2009

Address for submission: PD Dr. D. Hahnloser, Wissenschaftliche Kommission SALTC, Klinik für Viszeral- und Transplantationschirurgie, UniverstätsSpital Zürich, 8091 Zurich, e-mail: dieter.hahnloser@usz.ch

\section{Stiftung Eternit-Werke Schweiz}

\section{Unbürokratische Hilfe für Asbest-Opfer}

Die Eternit (Schweiz) AG unterhält eine Stiftung zur Linderung finanzieller Härtefälle bei Leidtragenden asbestbedingter Krankheiten. Das Unternehmen appelliert an die Ärzteschaft, Patienten aus dem Kreis der potentiell Begünstigten nicht nur der SUVA zu melden, sondern sie auch auf die Stiftung Eternit-Werke Schweiz aufmerksam zu machen.
Die schon in der Antike bekannte Mineralfaser Asbest war jahrzehntelang ein weltweit von Industrie und Baubranche hochgeschätzter Werkstoff. So war Asbest früher auch Bestandteil von Eternit, dem 1900 vom Österreicher Ludwig Hatschek erfundenen und patentierten Verbundwerkstoff. Erst Anfang der 1970er Jahre legten wissenschaftliche Studien zunehmend einen ursächlichen Zusammenhang zwischen Asbestfasern und bestimmten Krankheitsbildern fest. Das regelmässige Einatmen mikroskopisch kleiner Asbestfasern in Form eines von Auge nicht sichtbaren Feinstaubes kann das Entstehen von Asbestose oder eines Mesothelioms begünstigen. In der Schweiz führte diese Erkenntnis zum Entscheid der damaligen Eternit AG, auf die Verarbeitung von Asbest zu verzichten. Dennoch sind bis heute leider gegen 70 ehemalige Mitarbeiter an den Folgen asbestbedingter Berufskrankheiten gestorben.

Um Menschen, die durch asbestverursachte Krankheiten in finanzielle Schwierigkeiten gelangen, unbürokratisch zu helfen, hat die Eternit (Schweiz) AG im September 2007 die in Niederurnen domizilierte Stiftung EternitWerke Schweiz ins Leben gerufen. Leistungen der subsidiär wirkenden und mit insgesamt 2 Millionen Franken dotierten Stiftung Eternit-Werke Schweiz erhalten können Mitarbeiter der Eternit-Werke in Niederurnen (GL) und Payerne (VD) sowie deren hinterbliebenen nahen Angehörigen (Ehegatten, Lebenspartner und Kinder). Die Stiftung kann aber auch Personen begünstigen, die nie in diesen Eternit-Werken gearbeitet haben, deren Erkrankungen aber in einem ursächlichen Bezug zu den erwähnten Werken bzw. Werkstandorten stehen. Auch in diesem Fall gilt, dass hinterbliebene nahe Angehörige mit eingeschlossen sind.

Die Stiftung Eternit-Werke Schweiz ist auf dem Postweg (Giacomettistrasse 112, 7000 Chur) sowie via Internet erreichbar. Die in Deutsch, Französisch und Englisch gehaltene Homepage www.eternit-stiftung.ch informiert über die Stiftung und erlaubt das Herunterladen eines Anmeldeformulars.

Prof. Dr. med. Kaspar Rhyner, Stiftungsrat der Stiftung Eternit-Werke-Schweiz 